This item was submitted to Loughborough's Research Repository by the author.

Items in Figshare are protected by copyright, with all rights reserved, unless otherwise indicated.

\title{
The characterization of two-component (2+1)-dimensional integrable systems of hydrodynamic type
}

PLEASE CITE THE PUBLISHED VERSION

LICENCE

CC BY-NC-ND 4.0

\section{REPOSITORY RECORD}

Ferapontov, E.V., and Karima R. Khusnutdinova. 2019. "The Characterization of Two-component (2+1)dimensional Integrable Systems of Hydrodynamic Type”. figshare. https://hdl.handle.net/2134/300. 


\title{
The characterization of two-component (2+1)-dimensional integrable systems of hydrodynamic type
}

\author{
E.V. Ferapontov and K.R. Khusnutdinova * \\ Department of Mathematical Sciences \\ Loughborough University \\ Loughborough, Leicestershire LE11 3TU \\ United Kingdom \\ e-mails: \\ E.V.Ferapontov@lboro.ac.uk \\ K. Khusnutdinova@lboro.ac.uk
}

\begin{abstract}
We obtain the necessary and sufficient conditions for a two-component (2+1)-dimensional system of hydrodynamic type to possess infinitely many hydrodynamic reductions. These conditions are in involution, implying that the systems in question are locally parametrized by 15 arbitrary constants. It is proved that all such systems possess three conservation laws of hydrodynamic type and, therefore, are symmetrizable in Godunov's sense. Moreover, all such systems are proved to possess a scalar pseudopotential which plays the role of the 'dispersionless Lax pair'. We demonstrate that the class of two-component systems possessing a scalar pseudopotential is in fact identical with the class of systems possessing infinitely many hydrodynamic reductions, thus establishing the equivalence of the two possible definitions of the integrability. Explicit linearly degenerate examples are constructed.
\end{abstract}

MSC: 35L40, 35L65, 37K10.

Keywords: Multidimensional Systems of Hydrodynamic Type, Classification of Integrable Equations, Nonlinear Interactions of Simple Waves, Generalized Hodograph Method, Symmetrization, Pseudopotentials.

${ }^{*}$ On the leave from: Institute of Mechanics, Ufa Branch of the Russian Academy of Sciences, Karl Marx Str. 6, Ufa, 450000, Russia. 


\section{Introduction}

We consider the problem of the classification of $(2+1)$-dimensional integrable quasilinear systems

$$
\mathbf{u}_{t}+A(\mathbf{u}) \mathbf{u}_{x}+B(\mathbf{u}) \mathbf{u}_{y}=0
$$

where $t, x, y$ are independent variables, $\mathbf{u}$ is an $m$-component column vector and $A(\mathbf{u}), B(\mathbf{u})$ are $m \times m$ matrices. We assume that the system is strictly hyperbolic, that is, the generic matrix of the linear family $\lambda I_{m}+\mu A+B$ has $m$ distinct real eigenvalues. Following our recent paper [9], we call the system (1) integrable if it possesses 'sufficiently many' exact solutions of the form $\mathbf{u}=\mathbf{u}\left(R^{1}, \ldots, R^{n}\right)$ where the Riemann invariants $R^{1}, \ldots, R^{n}$ solve a pair of commuting diagonal systems

$$
R_{t}^{i}=\lambda^{i}(R) R_{y}^{i}, \quad R_{x}^{i}=\mu^{i}(R) R_{y}^{i}
$$

we emphasize that the number $n$ of Riemann invariants is allowed to be arbitrary. Solutions of this type, known as nonlinear interactions of $n$ planar simple waves, were discussed in a series of publications $[2,3,26,15]$. Later, they were investigated by Gibbons and Tsarev in the context of the dispersionless KP hierarchy [10, 11, 12, 13], see also [22], and the theory of Egorov's integrable hydrodynamic chains $[24,25]$.

We recall, see [28], that the requirement of the commutativity of the flows (2) is equivalent to the following restrictions on their characteristic speeds:

$$
\frac{\partial_{j} \lambda^{i}}{\lambda^{j}-\lambda^{i}}=\frac{\partial_{j} \mu^{i}}{\mu^{j}-\mu^{i}}, \quad i \neq j, \quad \partial_{j}=\partial / \partial_{R^{j}}
$$

(no summation!) Once these conditions are met, the general solution of (2) is given by the implicit 'generalized hodograph' formula [28],

$$
v^{i}(R)=y+\lambda^{i}(R) t+\mu^{i}(R) x, \quad i=1, \ldots, n,
$$

where $v^{i}(R)$ are characteristic speeds of the general flow commuting with (2), that is, the general solution of the linear system

$$
\frac{\partial_{j} v^{i}}{v^{j}-v^{i}}=\frac{\partial_{j} \lambda^{i}}{\lambda^{j}-\lambda^{i}}=\frac{\partial_{j} \mu^{i}}{\mu^{j}-\mu^{i}}
$$

Substituting $\mathbf{u}\left(R^{1}, \ldots, R^{n}\right)$ into (1) and using (2), one readily arrives at the equations

$$
\left(\lambda^{i} I_{m}+\mu^{i} A+B\right) \partial_{i} \mathbf{u}=0, \quad i=1, \ldots, n,
$$

implying that $\lambda^{i}$ and $\mu^{i}$ satisfy the dispersion relation

$$
\operatorname{det}\left(\lambda I_{m}+\mu A+B\right)=0 .
$$

Thus, the construction of nonlinear interactions of $n$ planar simple waves reduces to solving the equations (3), (6) for $\mathbf{u}(R), \lambda^{i}(R), \mu^{i}(R)$ as functions of the Riemann invariants $R^{1}, \ldots, R^{n}$. For $n \geq 3$ these equations are highly overdetermined and do not possess solutions in general. As demonstrated in [9], the requirement of the existence of nontrivial 3-component reductions is very restrictive and implies, in particular, the existence of $n$-component reductions for arbitrary $n$. We give the following 
Definition. The system (1) is said to be integrable if it possesses n-component reductions of the form (2) parametrized by $n$ arbitrary functions of a single argument.

We refer to [9] for the motivation and supporting examples.

Remark 1. In the case of linear systems (1), that is, in the case when both $A$ and $B$ are constant matrices, the equations (3) and (7) imply $\lambda_{j}^{i}=\mu_{j}^{i}=0$, so that $\lambda^{i}=\lambda^{i}\left(R^{i}\right), \mu^{i}=\mu^{i}\left(R^{i}\right)$. Moreover, as follows from (6), $\partial_{i} u=\xi_{i}\left(R^{i}\right)$ where $\xi_{i}\left(R^{i}\right)$ is the right eigenvector of the matrix $\lambda^{i} I_{m}+\mu^{i} A+B$. With the particular choice $\lambda^{i}=$ const, $\mu^{i}=$ const, $\xi_{i}=$ const the corresponding solutions represent the standard linear superposition of simple waves, $u=\sum f^{i}\left(x+\lambda^{i} t+\mu^{i} y\right) \xi_{i}$.

In Sect. 2 we derive the integrability conditions for the two-component system (1) assuming that the matrix $A$ is written in the diagonal form,

$$
\left(\begin{array}{c}
v \\
w
\end{array}\right)_{t}+\left(\begin{array}{cc}
a & 0 \\
0 & b
\end{array}\right)\left(\begin{array}{c}
v \\
w
\end{array}\right)_{x}+\left(\begin{array}{cc}
p & q \\
r & s
\end{array}\right)\left(\begin{array}{c}
v \\
w
\end{array}\right)_{y}=0
$$

such diagonalization is always possible in the two-component situation. These conditions constitute a complicated overdetermined system (13) - (17) of second order PDEs for $a, b, p, q, r, s$ as functions of $v, w$, which is in involution; a simple analysis shows that the class of integrable two-component systems is locally parametrised by 15 arbitrary constants.

Remark 2. In principle, the method described in Sect. 2 allows one to derive the integrability conditions in arbitrary coordinates, however, the formulasbecome extremely complicated. We were not able to find an invariant 'tensor' formulation of the integrability conditions so far.

We prove (Theorem 1 of Sect. 3) that an arbitrary two-component system (8) satisfying the integrability conditions possesses three conservation laws of hydrodynamic type and, thus, is symmetrizable in Godunov's sense [14].

In Sect. 4 we demonstrate that all two-component integrable systems possess scalar pseudopotentials of the form

$$
\psi_{t}=f\left(\psi_{y}, v, w\right), \quad \psi_{x}=g\left(\psi_{y}, v, w\right) .
$$

According to the philosophy of [29], this indicates that (2+1)-dimensional integrable systems of hydrodynamic type can be obtained as dispersionless limits from the appropriate $(2+1)$ dimensional integrable soliton equations (possibly, nonlocal, differential-difference, etc). The corresponding pseudopotentials are quasiclassical limits of the associated linear Lax operators. The construction of the 'solitonic prototype' was sketched in the case when the dependence of $f$ and $g$ on $\psi_{y}$ is rational (trigonometric), leading to differential (difference) soliton equations. We prove (Theorem 2 of Sect. 4) that the requirement of the existence of a scalar pseudopotential is, in fact, necessary and sufficient for the existence of the infinity of hydrodynamic reductions. This establishes the equivalence of the two approaches to integrability of $(2+1)$-dimensional hydrodynamic type systems. The quasi-classical $\bar{\partial}$-dressing approach to the solution of $(2+1)$ dimensional dispersionless systems based on the pseudopotentials of the above type was proposed in the series of recent publications $[16,17,18,19,1]$. It is not completely clear at the moment how exact solutions describing nonlinear interactions of planar simple waves fit into this scheme.

Some explicit examples where the matrix $A$ is linearly degenerate (that is, $a_{v}=b_{w}=0$ ), are discussed in Sect. 5. These include a remarkable case where both matrices $A$ and $B$, as well as arbitrary linear combinations thereof, are linearly degenerate. 
We conclude this introduction by listing some known examples of two-component integrable systems written in the form (8).

Example 1. Let us consider the system

$$
v_{t}+\frac{1}{v+w} v_{x}-\frac{1}{v+w} w_{y}=0, \quad w_{t}-\frac{1}{v+w} w_{x}+\frac{1}{v+w} v_{y}=0
$$

here $a=\frac{1}{v+w}, b=-\frac{1}{v+w}$, etc. Introducing the variables $m=v+w, n=v-w$, one can rewrite these equations in the form

$$
\left(\partial_{x}+\partial_{y}\right) n+\frac{1}{2} \partial_{t} m^{2}=0, \quad \partial_{t} n+\left(\partial_{x}-\partial_{y}\right) \ln m=0
$$

leading, upon cross-differentiation, to the Boyer-Finley equation for $m^{2}=(v+w)^{2}$ :

$$
\partial_{t}^{2} m^{2}=\left(\partial_{x}^{2}-\partial_{y}^{2}\right) \ln m^{2} .
$$

The Boyer-Finley equation is known to be integrable, its hydrodynamic reductions were investigated, e.g., in [7].

Example 2. A closely related example is

$$
v_{t}+\frac{1}{v+w} v_{x}+\frac{1}{v+w} \sqrt{\frac{v}{w}} w_{y}=0, \quad w_{t}-\frac{1}{v+w} w_{x}+\frac{1}{v+w} \sqrt{\frac{w}{v}} v_{y}=0 ;
$$

notice that the characteristic speeds $a$ and $b$ are the same as in the previous example! In the new variables $m=v-w, n=2 \sqrt{v w}$, this system reduces to

$$
m_{t}+\frac{m m_{x}+n n_{x}}{m^{2}+n^{2}}+\frac{m n_{y}-n m_{y}}{m^{2}+n^{2}}=0, \quad n_{t}+\frac{n m_{x}-m n_{x}}{m^{2}+n^{2}}+\frac{m m_{y}+n n_{y}}{m^{2}+n^{2}}=0 ;
$$

in this form it appeared in out recent paper [9]. It was demonstrated, in particular, that the expression $\rho^{2}=m^{2}+n^{2}=(v+w)^{2}$ satisfies another version of the Boyer-Finley equation,

$$
\partial_{t}^{2} \rho^{2}=\left(\partial_{x}^{2}+\partial_{y}^{2}\right) \ln \rho^{2},
$$

corresponding to different signature.

Example 3. Here both matrices $A$ and $B$, as well as arbitrary linear combinations thereof, are linearly degenerate:

$$
v_{t}+w v_{x}+\frac{1}{w-v}\left(v_{y}+w_{y}\right)=0, \quad w_{t}+v w_{x}+\frac{1}{v-w}\left(v_{y}+w_{y}\right)=0 .
$$

Introducing the variables $m=v+w, n=v w$, one can rewrite these equations as

$$
m_{t}+n_{x}=0, \quad n_{t}+m n_{x}-n m_{x}+m_{y}=0 .
$$

This system was thoroughly investigated in [24], see also [23, 27].

Example 4. We also looked at the integrable systems (8) whose chararacteristic speeds $a$ and $b$ are of the form $a=v+w+\epsilon v, b=v+w+\epsilon w, \epsilon=$ const. The analysis showed that the only possible values for $\epsilon$ are $\epsilon=-1$ and $\epsilon=-2$. In the first case the matrix $A$ is linearly degenerate, see Sect. 4 for the general form of the corresponding matrix $B$. In the case $\epsilon=-2$ we obtained the system

$$
v_{t}+(v-w) v_{x}+w_{y}=0, \quad w_{t}+(w-v) w_{x}+v_{y}=0,
$$

which is yet another first order form of the Boyer-Finley equation, indeed, this system reduces to that from the Example 1 after a simple change of variables $w \rightarrow-w, t \leftrightarrow x$. 


\section{Derivation of the integrability conditions}

The integrability conditions can be obtained as follows. Looking for reductions of the system (8) in the form $v=v\left(R^{1}, \ldots, R^{n}\right), w=w\left(R^{1}, \ldots, R^{n}\right)$ where the Riemann invariants satisfy the equations (2), and substituting into (8), one arrives at

$$
\left(\lambda^{i}+a \mu^{i}+p\right) \partial_{i} v+q \partial_{i} w=0, \quad r \partial_{i} v+\left(\lambda^{i}+b \mu^{i}+s\right) \partial_{i} w=0,
$$

(no summation!) so that $\lambda^{i}$ and $\mu^{i}$ satisfy the dispersion relation

$$
\left(\lambda^{i}+a \mu^{i}+p\right)\left(\lambda^{i}+b \mu^{i}+s\right)=q r .
$$

We assume that the dispersion relation defines an irreducible conic, that is, $a \neq b, r \neq 0, q \neq 0$. Notice that these conditions are equivalent to the requirement $r k[A, B]=2$. Setting $\partial_{i} v=\varphi^{i} \partial_{i} w$ one obtains the following expressions for $\lambda^{i}$ and $\mu^{i}$ in terms of $\varphi^{i}$,

$$
\lambda^{i}=\frac{a r\left(\varphi^{i}\right)^{2}+(a s-b p) \varphi^{i}-b q}{(b-a) \varphi^{i}}, \quad \mu^{i}=\frac{r\left(\varphi^{i}\right)^{2}+(s-p) \varphi^{i}-q}{(a-b) \varphi^{i}},
$$

which define a rational parametrization of the dispersion relation. The compatibility conditions of the equations $\partial_{i} v=\varphi^{i} \partial_{i} w$ imply

$$
\partial_{i} \partial_{j} w=\frac{\partial_{j} \varphi^{i}}{\varphi^{j}-\varphi^{i}} \partial_{i} w+\frac{\partial_{i} \varphi^{j}}{\varphi^{i}-\varphi^{j}} \partial_{j} w
$$

while the commutativity conditions (3) lead to the expressions for $\partial_{j} \varphi^{i},(i \neq j)$, in the form

$$
\partial_{j} \varphi^{i}=(\ldots) \partial_{j} w ;
$$

here dots denote a rational expression in $\varphi^{i}, \varphi^{j}$ whose coefficients depend on $a, b, p, q, r, s$ and the first derivatives thereof. We do not write them out explicitly due to their complexity. To manipulate with these expressions we used symbolic computations (Mathematica 5.0). Substituting the expressions for $\partial_{j} \varphi^{i}$ into (10) one obtains

$$
\partial_{i} \partial_{j} w=(\ldots) \partial_{i} w \partial_{j} w
$$

where, again, dots denote a rational expression in $\varphi^{i}, \varphi^{j}$. One can see that the compatibility conditions of the equations (11), that is, $\partial_{k} \partial_{j} \varphi^{i}-\partial_{j} \partial_{k} \varphi^{i}=0$, are of the form $P \partial_{j} w \partial_{k} w=0$, where $P$ is a complicated rational expression in $\varphi^{i}, \varphi^{j}, \varphi^{k}$ whose coefficients depend on partial derivatives of $a, b, p, q, r, s$ up to second order (to obtain the integrability conditions it suffices to consider 3 -component reductions setting $i=1, j=2, k=3$ ). Requiring that $P$ vanishes identically we obtain the expressions for all second partial derivatives of the potentials $a, b, p, s$, as well as three relations among the second partial derivatives of $q$ and $r$. Similarly, the compatibility conditions of the equations (12), that is, $\partial_{k}\left(\partial_{i} \partial_{j} w\right)-\partial_{j}\left(\partial_{i} \partial_{k} w\right)=0$, take the form $Q \partial_{i} w \partial_{j} w \partial_{k} w=0$ where, again, $Q$ is a rational expression in $\varphi^{i}, \varphi^{j}, \varphi^{k}$. Equating $Q$ to zero one obtains (modulo conditions obtained on the previous step) the expressions for mixed partial derivatives $q_{v w}$ and $r_{v w}$. The resulting set of the integrability conditions looks as follows.

\section{Equations for $a$ :}

$$
\begin{aligned}
a_{v v} & =\frac{q a_{v} b_{v}+2 q a_{v}^{2}+(s-p) a_{v} a_{w}-r a_{w}^{2}}{(a-b) q}+\frac{a_{v} r_{v}}{r}+\frac{2 a_{v} p_{w}-a_{w} p_{v}}{q}, \\
a_{v w} & =a_{v} \frac{a_{w}+b_{w}}{a-b}+a_{v}\left(\frac{q_{w}}{q}+\frac{r_{w}}{r}\right), \\
a_{w w} & =\frac{q a_{v} b_{v}+(s-p) a_{v} b_{w}+r a_{w}^{2}}{(a-b) r}+\frac{a_{v} s_{w}}{r}+\frac{a_{w} q_{w}}{q} .
\end{aligned}
$$




\section{Equations for $b$ :}

$$
\begin{aligned}
b_{v v} & =\frac{r a_{w} b_{w}+(p-s) a_{v} b_{w}+q b_{v}^{2}}{(b-a) q}+\frac{b_{w} p_{v}}{q}+\frac{b_{v} r_{v}}{r} \\
b_{v w} & =b_{w} \frac{a_{v}+b_{v}}{b-a}+b_{w}\left(\frac{q_{v}}{q}+\frac{r_{v}}{r}\right), \\
b_{w w} & =\frac{r a_{w} b_{w}+2 r b_{w}^{2}+(p-s) b_{v} b_{w}-q b_{v}^{2}}{(b-a) r}+\frac{b_{w} q_{w}}{q}+\frac{2 b_{w} s_{v}-b_{v} s_{w}}{r} .
\end{aligned}
$$

\section{Equations for $p$ :}

$$
\begin{aligned}
p_{v v}= & 2 \frac{r\left(a_{v} b_{w}-a_{w} b_{v}\right)+(s-p) a_{v} b_{v}}{(a-b)^{2}}+\frac{r_{v} p_{v}}{r}+\frac{p_{v} p_{w}}{q}+ \\
& \frac{\frac{r}{q}\left(2 q_{v} a_{w}-2 a_{v} q_{w}+a_{w} p_{w}\right)-b_{v} p_{v}+2 r_{v} a_{w}-2 a_{v}\left(s_{v}+p_{v}+r_{w}\right)+\frac{p-s}{q}\left(2 p_{v} a_{w}-a_{v} p_{w}\right)}{b-a} \\
p_{v w}= & 2(s-p) \frac{a_{v} b_{w}}{(a-b)^{2}}-\frac{b_{w} p_{v}+\left(2 s_{w}+p_{w}\right) a_{v}}{b-a}+p_{v}\left(\frac{q_{w}}{q}+\frac{r_{w}}{r}\right), \\
p_{w w}= & 2 \frac{q\left(a_{w} b_{v}-a_{v} b_{w}\right)+(s-p) a_{w} b_{w}}{(a-b)^{2}}+\frac{(p-s) b_{w} p_{v}-q b_{v} p_{v}-2 r s_{w} a_{w}-r a_{w} p_{w}}{(b-a) r}+ \\
& \frac{p_{v} s_{w}}{r}+\frac{q_{w} p_{w}}{q} .
\end{aligned}
$$

\section{Equations for $s$ :}

$$
\begin{aligned}
s_{v v}= & 2 \frac{r\left(a_{w} b_{v}-a_{v} b_{w}\right)+(p-s) a_{v} b_{v}}{(a-b)^{2}}+\frac{(s-p) a_{v} s_{w}-r a_{w} s_{w}-2 q p_{v} b_{v}-q b_{v} s_{v}}{(a-b) q}+ \\
& \frac{p_{v} s_{w}}{q}+\frac{r_{v} s_{v}}{r}, \\
s_{v w}= & 2(p-s) \frac{a_{v} b_{w}}{(a-b)^{2}}-\frac{a_{v} s_{w}+\left(2 p_{v}+s_{v}\right) b_{w}}{a-b}+s_{w}\left(\frac{q_{v}}{q}+\frac{r_{v}}{r}\right), \\
s_{w w}= & 2 \frac{q\left(a_{v} b_{w}-a_{w} b_{v}\right)+(p-s) a_{w} b_{w}}{(a-b)^{2}}+\frac{q_{w} s_{w}}{q}+\frac{s_{v} s_{w}}{r}+ \\
& \frac{\frac{q}{r}\left(2 r_{w} b_{v}-2 b_{w} r_{v}+b_{v} s_{v}\right)-a_{w} s_{w}+2 q_{w} b_{v}-2 b_{w}\left(p_{w}+s_{w}+q_{v}\right)+\frac{s-p}{r}\left(2 s_{w} b_{v}-b_{w} s_{v}\right)}{a-b} .
\end{aligned}
$$




\section{Equations for $q$ and $r$ :}

$$
\begin{aligned}
q r_{v v}+r q_{v v}= & 2(p-s) \frac{(p-s) a_{w} b_{w}+q\left(a_{v} b_{w}-a_{w} b_{v}\right)}{(a-b)^{2}}+q \frac{r_{v}}{r} \frac{q b_{v}+(s-p) b_{w}}{a-b}+ \\
& (s-p) \frac{2 a_{w} s_{w}+2 b_{w} p_{w}+b_{w} q_{v}}{a-b}+r \frac{\left(a_{w}-2 b_{w}\right) q_{w}}{a-b}+ \\
& q \frac{a_{w} r_{w}+b_{v}\left(2 p_{w}+2 s_{w}+q_{v}\right)-2 b_{w}\left(r_{w}+p_{v}+s_{v}\right)}{a-b}+ \\
& \frac{r}{q} q_{w}^{2}+\frac{q}{r} s_{w} r_{v}-q_{w} r_{w}+s_{w}\left(2 p_{w}+q_{v}\right), \\
q_{v w}= & (s-p) \frac{q a_{v} b_{v}+(s-p) a_{v} b_{w}+r a_{w} b_{w}}{r(a-b)^{2}}+\frac{q_{v} q_{w}}{q}+\frac{p_{v} s_{w}}{r}+ \\
& \frac{a_{v}\left(r q_{w}+q r_{w}\right)+(s-p)\left(a_{v} s_{w}+b_{w} p_{v}\right)+r a_{w} s_{w}+q p_{v} b_{v}}{r(a)}, \\
r_{v w}= & (p-s) \frac{r a_{w} b_{w}+(p-s) a_{v} b_{w}+q a_{v} b_{v}}{q(a-b)^{2}}+\frac{r_{v} r_{w}}{r}+\frac{p_{v} s_{w}}{q}+ \\
& \frac{b_{w}\left(r q_{v}+q r_{v}\right)+(p-s)\left(a_{v} s_{w}+b_{w} p_{v}\right)+r a_{w} s_{w}+q p_{v} b_{v}}{q(b-a)}, \\
q r_{w w}+r q_{w w}= & 2(s-p) \frac{(s-p) a_{v} b_{v}+r\left(a_{v} b_{w}-a_{w} b_{v}\right)}{(a-b)^{2}}+r \frac{q_{w}}{q} \frac{r a_{w}+(p-s) a_{v}}{b-a}+ \\
& (p-s) \frac{2 b_{v} p_{v}+2 a_{v} s_{v}+a_{v} r_{w}}{b-a}+q \frac{\left(b_{v}-2 a_{v}\right) r_{v}}{b-a}+ \\
& r \frac{b_{v} q_{v}+a_{w}\left(2 s_{v}+2 p_{v}+r_{w}\right)-2 a_{v}\left(q_{v}+s_{w}+p_{w}\right)}{b-a}+ \\
& \frac{q}{r} r_{v}^{2}+\frac{r}{q} p_{v} q_{w}-r_{v} q_{v}+p_{v}\left(2 s_{v}+r_{w}\right) ;
\end{aligned}
$$

notice that there are only two relations among the second derivatives $q_{v v}, r_{v v}, q_{w w}, r_{w w}$. These formulas are completely symmetric under the identification $v \leftrightarrow w, a \leftrightarrow b, p \leftrightarrow s, q \leftrightarrow r$. It can be verified that the equations (13) - (17) are in involution and their general solution depends, modulo the coordinate transformations $v=\varphi(\tilde{v}), w=\psi(\tilde{w})$, on 15 arbitrary constants. Thus, we have established the existence of a 15-parameter family of integrable systems of the form (8).

Once the integrability conditions (13) - (17) are satisfied, the general solution of the involutive system (11), (12) for $\varphi^{i}$ and $w$ will depend on $2 n$ arbitrary functions of a single argument (indeed, one can formulate the Goursat problem for this system specifying $\varphi^{i}$ along the $R^{i}$ coordinate line and specifying the restriction of $w$ to each of the coordinate lines). This has to be considered up to reparametrizations of the form $R^{i} \rightarrow f^{i}\left(R^{i}\right)$. Thus, the general $n$ component reduction depends on $n$ essential functions of a single argument. This justifies the definition of the integrability given in the Introduction. The system (11), (12) governing $n$ component reductions will be called the generalized Gibbons-Tsarev system (it was derived by Gibbons and Tsarev [12] in the context of the dispersionless KP equation).

Remark. Rewriting the equations $(13)_{2}$ and $(14)_{2}$ in the form $d \ln (q r)=\Omega$, where

$$
\Omega=\left(\frac{b_{v w}}{b_{w}}+\frac{a_{v}+b_{v}}{a-b}\right) d v+\left(\frac{a_{v w}}{a_{v}}+\frac{a_{w}+b_{w}}{b-a}\right) d w,
$$


(we assume $a_{v} \neq 0, b_{w} \neq 0$ ), one obtains the condition $d \Omega=0$ which involves the matrix $A$ only. Obviously, the same condition holds for an arbitrary matrix in the linear pencil $\alpha A+\beta B$ (written in the diagonal form). The object $d \Omega$ first appeared in $[5,6]$ as one of the basic reciprocal invariants of two-component hydrodynamic type systems.

\section{Conservation laws}

In this section we prove the following

Theorem 1 Any two-component (2+1)-dimensional system of hydrodynamic type which passes the integrability test necessarily possesses three conservation laws of hydrodynamic type and, hence, is symmetrizable in Godunov's sense [14].

This explains the observation made in our recent publication [9]. To obtain the proof we first transform the system into the form (8). Looking for conservation laws in the form

$$
h(v, w)_{t}+g(v, w)_{x}+f(v, w)_{y}=0,
$$

one readily obtains

$$
g_{v}=a h_{v}, \quad g_{w}=b h_{w}
$$

and

$$
f_{v}=p h_{v}+r h_{w}, \quad f_{w}=q h_{v}+s h_{w} .
$$

The consistency condition $g_{v w}=g_{w v}$ implies

$$
h_{v w}=\frac{a_{w}}{b-a} h_{v}+\frac{b_{v}}{a-b} h_{w},
$$

while the consistency condition $f_{v w}=f_{w v}$ results in

$$
\begin{gathered}
p_{w} h_{v}+p\left(\frac{a_{w}}{b-a} h_{v}+\frac{b_{v}}{a-b} h_{w}\right)+r_{w} h_{w}+r h_{w w}= \\
s_{v} h_{w}+s\left(\frac{a_{w}}{b-a} h_{v}+\frac{b_{v}}{a-b} h_{w}\right)+q_{v} h_{v}+q h_{v v} .
\end{gathered}
$$

The last formula can be rewritten in the form

$$
\begin{aligned}
& h_{v v}=\frac{1}{q}\left(\frac{s-p}{a-b} a_{w}+p_{w}-q_{v}\right) h_{v}+\frac{l}{q}, \\
& h_{w w}=\frac{1}{r}\left(\frac{s-p}{a-b} b_{v}+s_{v}-r_{w}\right) h_{w}+\frac{l}{r},
\end{aligned}
$$

where the equations for the auxiliary variable $l$ can be obtained from the compatibility conditions $\left(h_{v v}\right)_{w}=\left(h_{v w}\right)_{v}$ and $\left(h_{w w}\right)_{v}=\left(h_{v w}\right)_{w}$ :

$$
\begin{gathered}
l_{v}=\left(\frac{r_{v}}{r}+\frac{b_{v}}{a-b}\right) l-\frac{2 b_{v} p_{v}(b-a)+2 a_{v} b_{v}(p-s)+4 r a_{w} b_{v}-r a_{v} b_{w}}{(a-b)^{2}} h_{w}- \\
\frac{a_{v} b_{v} q+a_{w} b_{w} r+(a-b) r a_{w} \frac{q w}{q}+\left(a_{w} b_{v}-a_{v} b_{w}\right)(p-s)+(b-a) a_{w}\left(s_{v}-r_{w}\right)+(a-b) a_{v} s_{w}-r a_{w}^{2}}{(a-b)^{2}} h_{v}, \\
l_{w}=\left(\frac{q_{w}}{q}+\frac{a_{w}}{b-a}\right) l-\frac{2 a_{w} s_{w}(a-b)+2 a_{w} b_{w}(s-p)+4 q a_{w} b_{v}-q a_{v} b_{w}}{(a-b)^{2}} h_{v}- \\
\frac{a_{v} b_{v} q+a_{w} b_{w} r+(b-a) q b_{v} \frac{r_{v}}{r}+\left(a_{w} b_{v}-a_{v} b_{w}\right)(s-p)+(a-b) b_{v}\left(p_{w}-q_{v}\right)+(b-a) b_{w} p_{v}-q b_{v}^{2}}{(a-b)^{2}} h_{w} .
\end{gathered}
$$


One can verify that the compatibility conditions $l_{v w}=l_{w v}$ are satisfied identically by virtue of (13)-(17). Thus, the system of equations (18), (19) and (20) for conservation laws is in involution and its solution space is three-dimensional.

\section{Pseudopotentials}

In this section we prove that any integrable system (8) possesses a scalar pseudopotential depending, in some cases, on the auxiliary parameter $\lambda$. We begin with some supporting examples.

Example 5. The linearly degenerate system

$$
m_{t}+n_{x}=0, \quad n_{t}+m n_{x}-n m_{x}+m_{y}=0
$$

from the Example 3 possesses the pseudopotential

$$
\psi_{t}=-(\lambda+m) \psi_{x}, \quad \psi_{y}=\left(\lambda^{2}+\lambda m+n\right) \psi_{x} ;
$$

we emphasize that the parameter $\lambda$ is essential here, allowing one to recover the full system for $m, n$ from the consistency condition $\psi_{t y}=\psi_{y t}$.

Example 6. The dispersionless KP equation, $\left(u_{t}-u u_{x}\right)_{x}=u_{y y}$, rewritten in the twocomponent form

$$
u_{y}=w_{x}, \quad w_{y}=u_{t}-u u_{x}
$$

possesses the pseudopotential

$$
\psi_{t}=\frac{1}{3} \psi_{x}^{3}+u \psi_{x}+w, \quad \psi_{y}=\frac{1}{2} \psi_{x}^{2}+u,
$$

see [29].

Example 7. The Boyer-Finley equation, $u_{t t}=(\ln u)_{x y}$, rewritten in the 2-component form

$$
u_{t}=w_{y}, \quad w_{t}=u_{x} / u,
$$

possesses the pseudopotential

$$
\psi_{t}=\ln u-\ln \psi_{y}, \quad \psi_{x}=w-\frac{u}{\psi_{y}} .
$$

Further examples of integrable $(2+1)$-dimensional equations possessing pseudopotentials of the above type can be found in $[29,25,16]$. It is a remarkable fact that in all examples constructed in [25] the existence of such pseudopotentials manifests the equivalence of the corresponding $(2+1)$-dimensional system to a pair of commuting $(1+1)$-dimensional hydrodynamic chains.

In the general case of system (8) we look for a pseudopotential in the form

$$
\psi_{t}=f\left(\psi_{y}, v, w\right), \quad \psi_{x}=g\left(\psi_{y}, v, w\right) .
$$

Writing out the consistency condition $\psi_{t x}=\psi_{x t}$, expressing $v_{t}, w_{t}$ by virtue of (8) and equating to zero coefficients at $v_{x}, v_{y}, w_{x}, w_{y}$, one arrives at the following expressions for the first derivatives $f_{v}, f_{w}, f_{\xi}$ and $g_{\xi}$ (we adopt the notation $\xi \equiv \psi_{y}$ ):

$$
\begin{gathered}
f_{v}=-a g_{v}, \quad f_{w}=-b g_{w}, \\
f_{\xi}=\frac{b\left(p+r \frac{g_{w}}{g_{v}}\right)-a\left(s+q \frac{g_{v}}{g_{w}}\right)}{a-b},
\end{gathered}
$$


and

$$
g_{\xi}=\frac{s+q \frac{g_{v}}{g_{w}}-p-r \frac{g_{w}}{g_{v}}}{a-b} .
$$

The consistency conditions of the equations (22) imply the following expressions for the second partial derivatives $g_{v w}, g_{v v}, g_{w w}$ :

$$
\begin{gathered}
g_{v w}=\frac{a_{w}}{b-a} g_{v}+\frac{b_{v}}{a-b} g_{w}, \\
g_{v v}=\frac{g_{v}\left[g_{w}^{2}\left(r\left(b_{v}-a_{v}\right)+(a-b) r_{v}\right)+g_{v} g_{w}\left((a-b) p_{v}+(s-p) a_{v}-r a_{w}\right)+q a_{v} g_{v}^{2}\right]}{(a-b) r g_{w}^{2}}, \\
g_{w w}=\frac{g_{w}\left[g_{v}^{2}\left(q\left(a_{w}-b_{w}\right)+(b-a) q_{w}\right)+g_{v} g_{w}\left((b-a) s_{w}+(p-s) b_{w}-q b_{v}\right)+r b_{w} g_{w}^{2}\right]}{(b-a) q g_{v}^{2}} .
\end{gathered}
$$

The compatibility conditions of the equations (23), (24) for $g$, namely, the conditions $g_{\xi v v}=$ $g_{v v \xi}, g_{\xi v w}=g_{v w \xi}$, etc., are of the form $P\left(g_{v}, g_{w}\right)=0$, where $P$ denotes a rational expression in $g_{v}, g_{w}$ whose coefficients are functions of $a, b, p, q, r, s$ and their partial derivatives up to the second order. Equating all these expressions to zero (they are required to be zero identically in $g_{v}, g_{w}$ ), one obtains the set of conditions which are necessary and sufficient for the existence of pseudopotentials of the form (21). It is a truly remarkable fact that these conditions identically coincide with the integrability conditions (13) - (17). Thus, any system satisfying the integrability conditions (13) - (17) possesses pseudopotentials of the form (21) parametrized by 4 arbitrary integration constants, indeed, one can arbitrarily prescribe the values of $g, g_{v}, g_{w}$ and $f$ at any initial point; the rest is completely determined by the involutive system (23), (24) and (22). Notice, however, that the transformation $\psi \rightarrow \lambda \psi+\mu x+\nu y+\eta t$ allows one to eliminate all these constants in the general situation (see Example 5 where one of these constant survives and is essential).

We have established the following

Theorem 2 The class of two-component (2+1)-dimensional systems of hydrodynamic type possessing infinitely many hydrodynamic reductions coincides with the class of systems possessing a scalar pseudopotential of the form (21).

Remark. The pseudopotential (21) readily implies a pseudopotential for the corresponding generalized Gibbons-Tsarev system (11), (12). Indeed, differentiating the equations (21) by $y$ and introducing $\xi=\psi_{y}$, one obtains

$$
\xi_{t}=\partial_{y} f(\xi, v, w)=f_{\xi} \xi_{y}+f_{v} v_{y}+f_{w} w_{y}, \quad \xi_{x}=\partial_{y} g(\xi, v, w)=g_{\xi} \xi_{y}+g_{v} v_{y}+g_{w} w_{y} .
$$

Assuming now that $\xi, v, w$ are functions of $n$ Riemann invariants $R^{1}, \ldots, R^{n}$ which satisfy the equations (2), one arrives at

$$
\xi_{i} \lambda^{i}=f_{\xi} \xi_{i}+f_{v} v_{i}+f_{w} w_{i}, \quad \xi_{i} \mu^{i}=g_{\xi} \xi_{i}+g_{v} v_{i}+g_{w} w_{i}
$$

Substituting here $v_{i}=\varphi^{i} w_{i}$, the expressions (9) for $\lambda^{i}$ and $\mu^{i}$ in terms of $\varphi^{i}$ (see Sect. 2), and taking into account the formulae (22), (23), one ends up with

$$
\xi_{i}=\frac{(a-b) \varphi^{i}}{r \varphi^{i} / g_{v}-q / g_{w}} w_{i}
$$

Equations (25) define a scalar pseudopotential for the generalized Gibbons-Tsarev system (11), (12), that is, the consistency conditions of (25) imply the equations (11), (12). 


\section{$5 \quad$ Examples}

The equations (13) - (17) are particularly convenient to analyze when the matrix $A$ is given (we emphasize that $a$ and $b$ cannot be arbitrary). The corresponding matrix $B$ is then defined up to a natural equivalence

$$
B \rightarrow \mu B+\nu A+\eta I_{2}
$$

generated by a linear change of the independent variables in the equations (8): $\tilde{t}=t, \tilde{x}=x, \tilde{y}=$ $\mu y+\nu x+\eta t$; here $\mu, \nu, \eta$ are arbitrary constants. Moreover, one has a freedom of the coordinate transformations $v=\varphi(\tilde{v}), w=\psi(\tilde{w})$ preserving the diagonal form of $A$. These transformations do not change $a, b, p, s$ and transform $q$ and $r$ according to the formulas

$$
\tilde{q}=q \frac{\psi^{\prime}(\tilde{w})}{\varphi^{\prime}(\tilde{v})}, \quad \tilde{r}=r \frac{\varphi^{\prime}(\tilde{v})}{\psi^{\prime}(\tilde{w})} .
$$

The classification results presented below are carried out up to this natural equivalence.

In this section we concentrate on the case when the matrix $A$ is linearly degenerate, that is, $a_{v}=b_{w}=0$. There are three essentially different cases to consider:

$$
\text { Case 1: } A=\left(\begin{array}{cc}
w & 0 \\
0 & v
\end{array}\right) ; \quad \text { Case 2: } A=\left(\begin{array}{cc}
\alpha & 0 \\
0 & \beta
\end{array}\right) ; \quad \text { Case 3: } A=\left(\begin{array}{cc}
w & 0 \\
0 & \beta
\end{array}\right) \text {; }
$$

here $\alpha$ and $\beta$ are arbitrary constants. Notice that without any loss of generality one can set $\alpha=1, \beta=0$. Below we restrict ourselves to the symmetric cases 1 and 2 , and show that there is a multi-parameter freedom in the formulas for $B$.

Case 1. Substituting $a=w, b=v$ into the integrability conditions (13) - (17), one obtains the overdetermined system for $p, q, r, s$ which can be explicitly integrated (the integration is fairly straightforward so that we skip the details). Up to the equivalence mentioned above we have

$$
p=\frac{f(w)}{w-v}-\alpha w^{2}, \quad q=\frac{f(v)}{w-v}, \quad r=\frac{f(w)}{v-w}, \quad s=\frac{f(v)}{v-w}-\alpha v^{2},
$$

where $f$ is a cubic polynomial, $f(z)=\alpha z^{3}+\beta z^{2}+\gamma z+\delta$, and $\alpha, \beta, \gamma, \delta$ are arbitrary constants. A remarkable property of this example is that any matrix in the linear pencil $B+\mu A$ is also linearly degenerate. In the particular case $\alpha=\beta=\gamma=0, \delta=1$ one has

$$
v_{t}+w v_{x}+\frac{1}{w-v}\left(v_{y}+w_{y}\right)=0, \quad w_{t}+v w_{x}+\frac{1}{v-w}\left(v_{y}+w_{y}\right)=0 .
$$

This system possesses three conservation laws

$$
\begin{gathered}
(v+w)_{t}+(v w)_{x}=0 \\
\left(v^{2}+v w+w^{2}\right)_{t}+(v w(v+w))_{x}-(v+w)_{y}=0
\end{gathered}
$$

and

$$
\left(v^{3}+v^{2} w+v w^{2}+w^{3}\right)_{t}+\left(v w\left(v^{2}+v w+w^{2}\right)\right)_{x}-(v+w)_{y}^{2}=0 .
$$

Introducing the variables $m=v+w, n=v w$, one can rewrite this system as

$$
m_{t}+n_{x}=0, \quad n_{t}+m n_{x}-n m_{x}+m_{y}=0 .
$$


In this form it was thoroughly investigated in [24], see also [23].

Case 2. Here $a$ and $b$ are constants, $a \neq b$. The corresponding equations for $p, q, r, s$ take the form

$$
\begin{gathered}
p_{v v}=p_{v}\left(\frac{p_{w}}{q}+\frac{r_{v}}{r}\right), \quad p_{v w}=p_{v}\left(\frac{q_{w}}{q}+\frac{r_{w}}{r}\right), \quad p_{w w}=\frac{p_{w} q_{w}}{q}+\frac{s_{w} p_{v}}{r} \\
s_{v v}=\frac{s_{v} r_{v}}{r}+\frac{s_{w} p_{v}}{q}, \quad s_{v w}=s_{w}\left(\frac{q_{v}}{q}+\frac{r_{v}}{r}\right), \quad s_{w w}=s_{w}\left(\frac{s_{v}}{r}+\frac{q_{w}}{q}\right) \\
q r_{v v}+r q_{v v}=\frac{q r_{v}^{2}}{r}-q_{v} r_{v}+p_{v}\left(2 s_{v}+r_{w}\right)+\frac{r q_{w} p_{v}}{q} \\
q r_{w w}+r q_{w w}=\frac{r q_{w}^{2}}{q}-q_{w} r_{w}+s_{w}\left(2 p_{w}+q_{v}\right)+\frac{q r_{v} s_{w}}{r} \\
q_{v w}=\frac{q_{v} q_{w}}{q}+\frac{p_{v} s_{w}}{r}, \quad r_{v w}=\frac{r_{v} r_{w}}{r}+\frac{p_{v} s_{w}}{q} .
\end{gathered}
$$

These equations imply, in particular, that $\left(p_{v} / q r\right)_{w}=0,\left(s_{w} / q r\right)_{v}=0$ so that, after the appropriate reparametrization $v \rightarrow f(v), w \rightarrow g(w)$, one can set $p_{v}=s_{w}=q r\left(\operatorname{provided} p_{v} \neq 0, s_{w} \neq 0\right)$. With this simplification, the above equations reduce to

$$
p_{v}=q r, \quad p_{w}=q_{v}, \quad s_{v}=r_{w}, \quad s_{w}=q r,
$$

along with the following overdetermined system for $q$ and $r$ :

$$
\begin{aligned}
& q_{v v}=(q r)_{w}, \quad q_{v w}=\frac{q_{v} q_{w}}{q}+q^{2} r, \quad q_{w w}=\frac{q_{w}^{2}}{q}+2 q q_{v}-\frac{q_{w} r_{w}}{r}, \\
& r_{w w}=(q r)_{v}, \quad r_{v w}=\frac{r_{v} r_{w}}{r}+q r^{2}, \quad r_{v v}=\frac{r_{v}^{2}}{r}+2 r r_{w}-\frac{q_{v} r_{v}}{q} .
\end{aligned}
$$

This system is in involution with the general solution depending on 6 arbitrary constants. Equations for $q_{v w}$ and $r_{v w}$ yield the Liouville equation for $\ln (q r)$ and the linear wave equation for $\ln (q / r)$, implying the following functional ansatz for these variables:

$$
q=\frac{f^{\prime}(v)^{1 / 2} g^{\prime}(w)^{1 / 2}}{f(v)+g(w)} \frac{m(f(v))}{n(g(w))}, \quad r=\frac{f^{\prime}(v)^{1 / 2} g^{\prime}(w)^{1 / 2}}{f(v)+g(w)} \frac{n(g(w))}{m(f(v))} .
$$

Setting

$$
\left(f^{\prime}\right)^{3 / 2}=P(f), \quad\left(g^{\prime}\right)^{3 / 2}=Q(g),
$$

and substituting (27) into the remaining equations (26), we obtain the following four functionaldifferential equations for $P(f), Q(g), m(f), n(g)$ :

$$
\begin{gathered}
{\left[P^{\prime \prime}(f+g)^{2}-4 P^{\prime}(f+g)+6 P\right] m+} \\
{\left[4 P^{\prime}(f+g)^{2}-6 P(f+g)\right] m^{\prime}+3 P(f+g)^{2} m^{\prime \prime}=\left[2 Q^{\prime}(f+g)-6 Q\right] n,} \\
{\left[Q^{\prime \prime}(f+g)^{2}-4 Q^{\prime}(f+g)+6 Q\right] n+} \\
{\left[4 Q^{\prime}(f+g)^{2}-6 Q(f+g)\right] n^{\prime}+3 Q(f+g)^{2} n^{\prime \prime}=\left[2 P^{\prime}(f+g)-6 P\right] m,} \\
P m^{\prime}+Q^{\prime}(f+g) n^{\prime}+Q\left[(f+g) n^{\prime \prime}-n^{\prime}\right]=0, \\
Q n^{\prime}+P^{\prime}(f+g) m^{\prime}+P\left[(f+g) m^{\prime \prime}-m^{\prime}\right]=0 .
\end{gathered}
$$


These equations yield

$$
P(f)=\frac{\alpha f^{3}+\beta f^{2}+\gamma f+\delta}{m(f)}, \quad Q(g)=\frac{\alpha g^{3}-\beta g^{2}+\gamma g-\delta}{n(g)},
$$

where

$$
(\ln m)^{\prime}=\frac{A f}{\alpha f^{3}+\beta f^{2}+\gamma f+\delta}, \quad(\ln n)^{\prime}=-\frac{A g}{\alpha g^{3}-\beta g^{2}+\gamma g-\delta} .
$$

Here, $\alpha, \beta, \gamma, \delta$ and $A$ are arbitrary constants. If $A=0$ and $m=n=1$, then both $P$ and $Q$ are cubic polynomials in $f$ and $g$, implying that equations for $f$ and $g$ can be solved in terms of elliptic functions (this case was considered in [9]).

\section{Conclusion}

In this paper we gave the characterization of two-component $(2+1)$-dimensional integrable systems of hydrodynamic type, showing that

- there exists a 15-parameter family of such systems;

- all integrable systems are symmetrizable in Godunov's sense;

- the system is integrable iff it possesses a scalar pseudopotential.

We have also constructed nontrivial explicit examples of integrable two-component $(2+1)$ dimensional systems of hydrodynamic type for which one of the matrices of the system is linearly degenerate.

The important problem remaining is to clarify the differential geometry of the full set of integrability conditions (13) - (17) expressing them in invariant form in terms of the corresponding matrices $A$ and $B$.

\section{Acknowledgements}

We are grateful to M. Pavlov and V. Sokolov for stimulating discussions.

\section{References}

[1] L.V. Bogdanov, B.G. Konopelchenko and L. Martinez Alonso, Quasi-classical $\bar{\partial}$-method: Generating equations for dispersionless integrable hierarchies, arXiv:nlin.SI/0111062.

[2] M. Burnat, The method of Riemann invariants for multi-dimensional nonelliptic system, Bull. Acad. Polon. Sci. Sr. Sci. Tech. 17 (1969) 1019-1026.

[3] M. Burnat, The method of characteristics and Riemann's invariants for multidimensional hyperbolic systems, (Russian) Sibirsk. Mat. Z. 11 (1970) 279-309.

[4] B.A. Dubrovin and S.P. Novikov, Hydrodynamics of weakly deformed soliton lattices: differential geometry and Hamiltonian theory, Russian Math. Surveys, 44 , N6 (1989) 35-124. 
[5] E.V. Ferapontov, Reciprocal transformations and their invariants, Diff. Uravneniya, 25, no. 7 (1989) 1256-1265 (English translation in Differential Equations, 25, no. 7 (1989) 898-905).

[6] E.V. Ferapontov, Reciprocal autotransformations and hydrodynamic symmetries, Diff. Uravneniya, 27, no.7 (1991) 1250-1263 (English translation in Differential Equations, 27, no. 7 (1991) 885-895).

[7] E.V. Ferapontov, D.A. Korotkin and V.A. Shramchenko, Boyer-Finley equation and systems of hydrodynamic type, Class. Quantum Grav. 19 (2002) L1-L6.

[8] E.V. Ferapontov and M.V. Pavlov, Hydrodynamic reductions of the heavenly equation, Class. Quantum Grav. 20 (2003) 1-13.

[9] E.V. Ferapontov and K.R. Khusnutdinova, On the integrability of 2+1-dimensional quasilinear systems, nlin.SI/0305044; to appear in Comm. Math. Phys. (2003).

[10] J. Gibbons, Collisionless Boltzmann equations and integrable moment equations, Physica 3D (1981) 503-511.

[11] J. Gibbons and Y. Kodama, A method for solving the dispersionless KP hierarchy and its exact solutions. II, Phys. Lett. A 135, no. 3 (1989) 167-170.

[12] J. Gibbons and S. P. Tsarev, Reductions of the Benney equations, Phys. Lett. A 211 (1996) $19-24$.

[13] J. Gibbons and S. P. Tsarev, Conformal maps and reductions of the Benney equations, Phys. Lett. A 258 (1999) 263-271.

[14] S.K. Godunov, An interesting class of quasi-linear systems, Dokl. Akad. Nauk SSSR 139 (1961) 521-523.

[15] A. Grundland and R. Zelazny, Simple waves in quasilinear hyperbolic systems. I, II. Riemann invariants for the problem of simple wave interactions, J. Math. Phys. 24, no. 9 (1983) 2305-2328.

[16] B.G. Konopelchenko and L. Martinez Alonso, Nonlinear dynamics on the plane and integrable hierarchies of infinitesimal deformations, Stud. Appl. Math. 109, no. 4 (2002) 313-336; arXiv:nlin.SI/0201039.

[17] B.G. Konopelchenko and L. Martinez Alonso, Dispersionless scalar integrable hierarchies, Whitham hierarchy, and the quasiclassical $\bar{\partial}$-dressing method, J. Math. Phys. 43, no. 7 (2002) 3807-3823.

[18] B.G. Konopelchenko, L. Martinez Alonso and O. Ragnisco, The $\bar{\partial}$-approach to the dispersionless KP hierarchy, J. Phys. A 34, no. 47 (2001) 10209-10217.

[19] B.G. Konopelchenko and L. Martinez Alonso, $\bar{\partial}$-equations, integrable deformations of quasiconformal mappings and Whitham hierarchy, Phys. Lett. A 286, no. 2-3 (2001) 161-166.

[20] I.M. Krichever, The averaging method for two-dimensional "integrable" equations, Funct. Anal. Appl. 22, no. 3 (1988) 200-213. 
[21] I.M. Krichever, Spectral theory of two-dimensional periodic operators and its applications, Russian Math. Surveys 44, no. 2 (1989) 145-225.

[22] M. Manas, L. Martinez Alonso and E. Medina, Reductions and hodograph solutions of the dispersionless KP hierarchy, J. Phys. A: Math. Gen. 35 (2002) 401-417.

[23] L. Martinez Alonso and A.B. Shabat, Energy-dependent potentials revisited: A universal hierarchy of hydrodynamic type, Phys. lett. A 299, no. 4 (2002) 359-365.

[24] M.V. Pavlov, Integrable hydrodynamic chains, J. Math. Phys. 44, no. 9 (2003); nlin.SI/0301010.

[25] M.V. Pavlov, Classification of the integrable Egorov hydrodynamic chains, submitted to Theor. and Math. Phys. (2003).

[26] Z. Peradzyński, Nonlinear plane $k$-waves and Riemann invariants, Bull. Acad. Polon. Sci. Sr. Sci. Tech. 19 (1971) 625-632.

[27] A.B. Shabat and L. Martinez Alonso, On the prolongation of a hierarchy of hydrodynamic chains, to appear in Theor. Math. Phys. (2003).

[28] S.P. Tsarev, Geometry of hamiltonian systems of hydrodynamic type. Generalized hodograph method, Izvestija AN USSR Math. 54, no. 5 (1990) 1048-1068.

[29] E.V. Zakharov, Dispersionless limit of integrable systems in $2+1$ dimensions, in Singular Limits of Dispersive Waves, Ed. N.M. Ercolani et al., Plenum Press, NY, (1994) 165-174. 